\title{
Significance of electrodermal activity response in children with autism spectrum disorder
}

\author{
Awais Gul Airij ${ }^{1}$, Rubita Sudirman ${ }^{2}$, Usman Ullah Sheikh ${ }^{3}$, Lee Yoot Khuan ${ }^{4}$, Nor Aini Zakaria ${ }^{5}$ \\ ${ }^{1,2,3,5}$ Department of Electronics and Computer Engineering, Faculty of Engineering, \\ Universiti Teknologi Malaysia, Malaysia \\ ${ }^{4}$ Faculty of Electrical Engineering \& Computational Intelligence Detection RIG, \\ Pharmaceutical \& Lifesciences Communities of Research Universiti Teknologi MARA, Malaysia
}

\begin{tabular}{l}
\hline \hline Article Info \\
\hline Article history: \\
Received Jan 4, 2020 \\
Revised Mar 6, 2020 \\
Accepted Mar 22, 2020 \\
\hline
\end{tabular}

Keywords:

Autism spectrum disorder Autonomic nervous system Electrodermal activity Physiological signals Stress

\begin{abstract}
The human Autonomic Nervous System (ANS) controls the body's physiological responses, such as heart rate, electrodermal activity, temperature, and pupil diameter. The physiological responses are increased in the presence of stressing stimuli, and this is a typical ANS response. However, in the case of children with Autism Spectrum Disorder (ASD), they suffer from autonomic dysregulation as reported in the past owing to their atypical ANS response. This study investigated the ANS response of children with ASD and compared it with the response of normal children. EDA response datasets of 35 children with ASD and 55 normal children were acquired with the help of E4 wristband at a sampling rate of $4 \mathrm{~Hz}$. The signals were preprocessed to remove artefacts and noise and later compared. Furthermore, an SVM classifier was also used to classify the EDA response signals of normal children and children with ASD. The obtained results highlight that the ANS response of children with ASD is atypical as their EDA response is blunt and shows no significant tonic and phasic changes in EDA levels in the presence of stressing stimuli. In addition to that, an accuracy of $75 \%$ was obtained using the LF kernel of SVM classifier. The study further unfolds the hypoactive sympathetic response of children with ASD during a stressing event. Furthermore, this will help in future to anticipate the emotional responses of children with ASD such as anger, happiness, and anxiety.
\end{abstract}

Copyright (@ 2020 Institute of Advanced Engineering and Science. All rights reserved.

\section{Corresponding Author:}

Awais Gul Airij,

Department of Electronics and Computer Engineering,

Faculty of Engineering,

Universiti Teknologi Malaysia, Johor, Malaysia.

Email: gaawais2@live.utm.my

\section{INTRODUCTION}

The human body is made up of complex mechanisms in which it is difficult to identify a problematic state without a battery of tests. However, the body does show multiple indications in case of distress or alarming situations, and those indications are known as physiological signals [1,2] produced by the body's physiological processes. The human physiological signals include heart rate, electrodermal activity (EDA) response, body temperature, pupil diameter, brain waves, and respiration [1, 3]. The variations in the abovesaid physiological signals highlight some problematic state such as stress, anxiety, fear, or any other form of emotional arousal [4-6]. For instance, the variation in Electrocardiogram (ECG) signal provides meaningful information regarding the heart's functionality [7]. These physiological signals are also an indication of some underlying medical conditions, hence, making these signals interesting enough to be studied and scrutinized in order to understand them and prevent harmful outcomes. The human 
physiological responses are governed by the Sympathetic Nervous System (SNS) and Parasympathetic Nervous System (PNS) which are the branches of Autonomic Nervous System (ANS) [1, 5]. The role of SNS is to increase the rate of physiological responses in case of any alarming situation. At the same time, the PNS helps to regulate these rates and bringing the body back to homeostasis. This phenomenon is known as a normal or typical or regular autonomic nervous system response. On the other hand, when the rate of physiological signals decreases in the presence of a stressor instead of increasing, such phenomenon is known as abnormal or atypical autonomic nervous system response. Such person is referred to as suffering from autonomic dysregulation [8].

Among the many physiological signals, this research only focuses on the study of variation in EDA response of a person as an indicator of distress or anxiety. The EDA response, which is an involuntary function is the measure of changes in skin's electrical properties due to the presence of stressors or some underlying medical conditions $[9,10]$. The EDA response is categorized into two types, known as phasic change and tonic change. The tonic change, which is also known as SCL [11], refers to the smooth and gradual changes in the EDA response signal, which occur in the absence of stressing stimuli. On the other hand, the phasic change, which is also known as SCR [11], refers to the rapid/sudden changes in the EDA response, as shown in Figure 1. The highlighted/encircled areas in Figure 1 show phasic changes and the remaining parts of the graph show tonic changes of an EDA response signal. The physiological responses of special children are not well-studied previously hence, the role of ANS response in special children is not clear. Therefore, this study aims at developing an understanding of only one physiological response which is EDA of children with Autism Spectrum Disorder (ASD) and normal children. ASD is a neurological and developmental disorder which starts in the infant stages and remains throughout an individual's life [12].

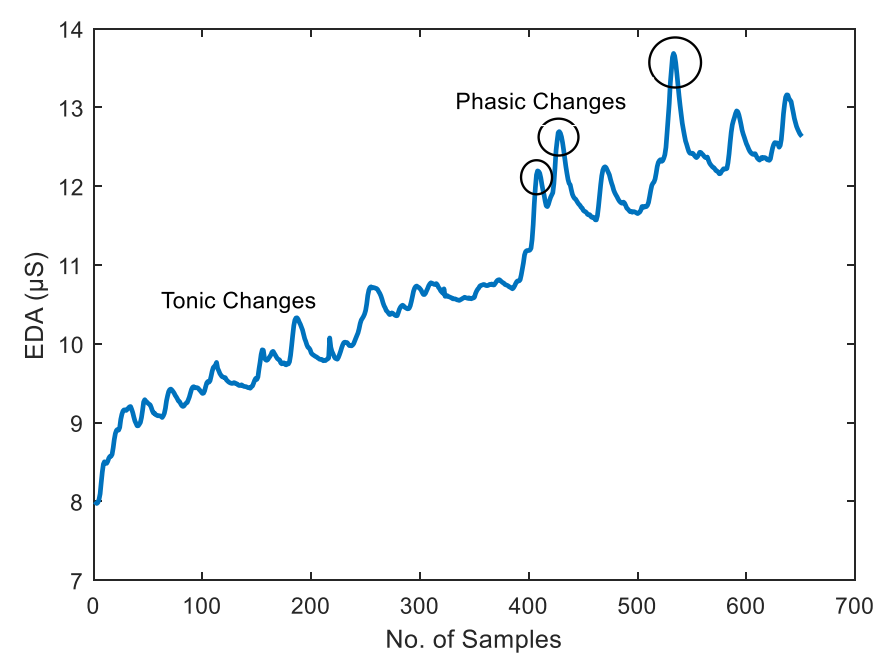

Figure 1. Phasic and tonic changes in an EDA response signal

In this regard, previously, many physiological signals have been studied for the purpose of stress, anxiety, and emotion recognition. For instance, in [13], only skin conductance was used to detect human stress along with two accelerometers and achieved an accuracy of 94.7\%. The experimental phase consisted of arithmetic problems as stressors which were meant to be solved by intended subjects, and decision tree classifier was used for this study. The accelerometers were used for differentiating between stress and physical activity however, the use of skin conductance as a significant stress indicator is still under scrutiny for children with ASD. Moreover, in [9] galvanic skin response sensor was used to measure skin conductance for the purpose of stress detection. The ADWIN and Fit methods were used in this study. It is a good research with a detailed explanation but lacks the element of wider subject range as the data was only collected from 5 subjects. Later in [14], a team of researchers monitored the cardiac activity of participants during some activities with the help of a wireless chest belt. The research monitored the cardiac response of 10 participants, including 5 children with ASD and 5 children with language disorder between the age of $2-5$ years. The research showed an increase in cardiac response during designed activities. In another research [15], the researchers collected two physiological signals named GSR and ECG for stress detection. The features were extracted using Fisher's discriminant criteria and Support Vector Machine (SVM) was used for stress classification. In the same line of research, [16] used heart rate, EDA response, and body 
temperature signals for classifying stress levels. The researcher used fuzzy logic, decision tree, Naïve Bayes, and K-NN classifiers and found that the fuzzy logic achieved an accuracy of $96 \%$, whereas the accuracy of other techniques was lower. The data was collected from 35 participants, and the arithmetic game was used to induce stress in participants.

Furthermore, in [17], changes in ANS were measured to determine stress among 15 children with ASD and 18 children without ASD. It was observed that cardiac activity was increased in children with ASD during baseline and anxiety induction test while the perspiration response was normal, and no change was found in skin temperature either. Whereas, in children without ASD, cardiac activity, perspiration, and temperature were only elevated during the experiment. Similarly, in [18], heart rate, GSR, and temperature signals were measured from both normal and autistic children. The research highlighted those autistic children had elevated GSR response in low-anxiety and high-anxiety situations as well. Another research [19] also acquired heart rate, EDA response, and body temperature signals for anxiety detection and reported a blunted EDA response in children with ASD during high-anxiety situations. They used the Stroop Colour Word (SCW) test for inducing stress in twenty-nine children, including 17 normal children and 12 children with ASD.

Based on the abovementioned previous works, it is evident that the EDA response is a significant indicator in applications such as stress monitoring, anxiety, and emotion recognition. However, if the autonomic dysregulation found in children with ASD is taken into consideration, it is questionable whether the EDA response of children with ASD is significant or not.

Therefore, the problem addressed in this research is to identify the significance of variation in EDA response signal of children with specials needs and normal children. Among special children, this paper specifically focuses on the EDA response of children with Autism Spectrum Disorder (ASD) as previous research suggests that they suffer from autonomic dysregulation $[8,10,17,19]$. The other objective of this work is to design a classification system capable of classifying the EDA responses of normal children and children with ASD. Furthermore, this paper also aims at resolving the conflict between previous research by comparing the EDA responses of children with ASD and normal children. This is because one research [18] states that children with ASD have increased EDA response during high-anxiety (stressing) situations whereas the other research [19] suggests that they have a blunt EDA response during these situations. Furthermore, previous research suggests that children with ASD have atypical ANS response [10] due to which their physiological response is different when compared with normal children. Hence, the questions investigated in this study are:

a) Whether the EDA response of children with ASD is a significant indicator of stress or not?

b) Do children with ASD have atypical ANS response or not?

The paper is organized in a manner that Section 1 provides a general introduction of the research along with the previous works, the problems addressed, and the objectives of this research. Section 2 covers the methodology, which includes the details of participants, stress-inducing test, data acquisition device, and the experimental setup. After that, the obtained results, discussions, and limitations of this study are mentioned in Section 3. Finally, a conclusion is made in Section 4.

\section{RESEARCH METHOD}

This section discusses the details regarding the participants, data acquisition device, and the steps taken to obtain the objectives of the study.

\subsection{Participants}

The EDA response signal data were collected from two groups of children with a total number of 90 participants. The first group $(\mathrm{G} 1)$ was of normal children $(n=55)$ within the age range of $4-12$ years and the second group $(\mathrm{G} 2)$ were of children with ASD $(n=35)$ within the age range of $4-12$ years. The children in the ASD group were selected from autistic centres and were diagnosed with ASD. The children in the normal group were selected from a school and did not have any mental or physical disabilities. All the parents of children were given consent forms prior to the data acquisition, which contained all the information regarding the study.

\subsection{Data acquisition device}

The E4 wristband by Empatica [20] was used for recording EDA response signals from all the participants. The device houses four sensors including photoplethysmography (PPG) sensor, EDA sensor, temperature sensor and a 3-axis accelerometer. The electrodes are silver coated and can be placed on a participant's wrist. The device can record up to 36+ hours [21] of data on a single charge, and the recorded data is automatically uploaded to Empatica's secure server. Furthermore, the data can also be accessed in real-time through its app available on Android and iOS. For this study, only EDA response data was obtained 
as the scope of this research is limited to studying the EDA response signals only. The EDA response signals were recorded at a non-customizable sampling frequency of $4 \mathrm{~Hz}$ with a range of $0.01-100 \mu$ Siemens. The previous research [22] suggests that the sampling frequency of 1 to $10 \mathrm{~Hz}$ is sufficient to obtain a GSR signal, hence, the chosen frequency of $4 \mathrm{~Hz}$ provides sufficient information.

\subsection{Stroop colour word test}

The Stroop Color and Word Test (SCWT) is a neuropsychological test extensively used for both experimental and clinical purposes [23]. The SCW test was used for inducing stress in the participants based on [23] and was divided into two segments; congruent and incongruent. The test consisted of three phases, Phase1, Phase2, and Phase3, and each phase comprised of 30 questions. The first phase was relatively easier. Then the second phase was designed to be a bit more difficult as it introduced the time limit while the third phase was designed to be very difficult. The first phase was of 5 minutes and the second phase was of 2.5 minutes with a time limit of $5 \mathrm{secs} /$ question. The third phase of the experiment was of 1 minute and 20 seconds with a time limit of 2 secs/question. The first two phases were based on the congruent segment of the SCW test, while the last phase was based on the incongruent segment. The SCW test was designed with the help of a software named "Wondershare Quiz Creator" as it offered all the necessary features required for this experiment. A snapshot of the quiz (experiment) is shown in Figure 2.

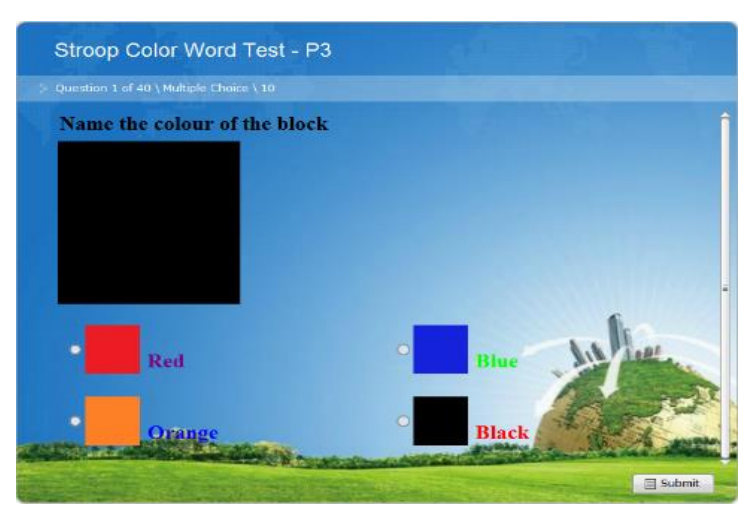

Figure 2. Sample of experiment

\subsection{Experimental procedure}

The procedure for data acquisition was completely non-invasive and took approximately 10 minutes per participant, and each participant was required to visit only once for this session. The detailed step by step procedure for data acquisition is shown below:

a. Participants were asked to sit in a calm environment.

b. Participants were asked to fill a form consisting of details such as random identification number, age, gender, and medical history/diagnosis/condition.

c. The E4 wristband was fastened to the participant's wrist in a manner that it was neither too tight nor too, loose, as shown in Figure 3.

d. For the baseline recordings, the participants will be asked to close their eyes and relax for 1 minute.

e. The participants were asked to solve the SCW test consisting of the abovementioned three phases.

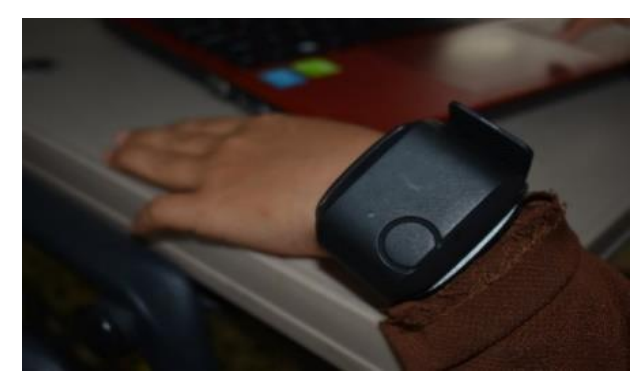

Figure 3. E4 wristband fastened to participant's wrist 


\subsection{Statistical analysis and classification}

The obtained signals of both groups G1 and G2 were statistically analysed with the help of t-test to identify the significance of the difference between the two sets. If the obtained value of the t-test is less than 0.05 , then it is said to be statistically significant.

Furthermore, a Support Vector Machine (SVM) based classifier was also implemented in MATLAB as previous research suggests that SVM classifiers provide better accuracy in similar cases [24, 25]. The classifier was used to differentiate between the EDA responses of the two groups with the help of training. The SVM classifier was trained with $80 \%$ of the data, and the remaining $20 \%$ of the data was used for testing. The prime issue when training a classifier is to find the most appropriate kernel function [24]. SVM offers multiple kernel functions among which Linear Function (LF) and Radial Basis Function (RBF) are utilized in this study. The equations for LF and RBF kernel functions of SVM are stated as:

$$
\begin{aligned}
& K(X, Y)=(X . Y+1) \\
& K(X, Y)=\exp \left(-\gamma\|X-Y\|^{2}\right)
\end{aligned}
$$

The compatibility of the kernel function is directly related to the efficiency of the SVM classifier, and it also affects the accuracy of the SVM classification system.

\section{RESULTS AND ANALYSIS}

As per the information provided in Section 2, the EDA sensor acquired data at a sampling rate of $4 \mathrm{~Hz}$, which means that it acquired 240 samples/minute. In this study, the EDA response signals were recorded for 8 minutes on average. Hence, the obtained total number of samples was 1920. Among them, only 1500 samples of EDA response data were analyzed. The remaining 420 samples were removed due to device setup, noise, and motion artefacts. The elimination and selection of samples are shown in Figure 4. The average of all 55 datasets from G1 and all 35 datasets from G2 was taken according to the phases: P1, P2, and P3 as shown in Figure 5. Each dataset contained 1500 samples, and each phase consisted of 500 samples. The average values show the average EDA response level, highlighting that the EDA values of G1 are increasing in accordance with the phases, while the EDA values of G2 show a little to no change with the increase in phases.

Along with that, for simplifying the analysis, the arithmetic mean $(\mu)$, standard deviation $(\sigma)$, and variance $\left(\sigma^{2}\right)$ of only ten participants including five from G1 and five from G2, were also calculated as shown in Table 1. Mean, Standard Deviation, and Variance of EDA response signals. Furthermore, all the signals were preprocessed by applying a moving average to remove the noise artefacts with a smoothing factor of 0.01. The subsequent subsections show the obtained results and discussion regarding the objectives mentioned in Section 1.

The EDA responses of G1 were compared with the EDA responses of G2, and all showed similar results. For the ease of understanding, the comparison of only 2 signals is shown in Figure 8, and among them, one signal is taken from G1 and compared with a signal of G2. It can be clearly seen in Figure 8 that the EDA response of G1 is increasing during the experiment with the increase in the difficulty of the SCW test. However, in the case of G2, the graph shows hypoactivity throughout the experiment. A similar case of hypoactivity of EDA response of sample from G2 can be witnessed in Figure 8 where the EDA response is blunt over time, and no significant phasic and tonic changes can be seen during the experiment. On the other hand, the sample from G1 is increasing over time. Moreover, after performing the statistical analysis, the research obtained a t-test value of 0.0254 , which shows that it is statistically significant.

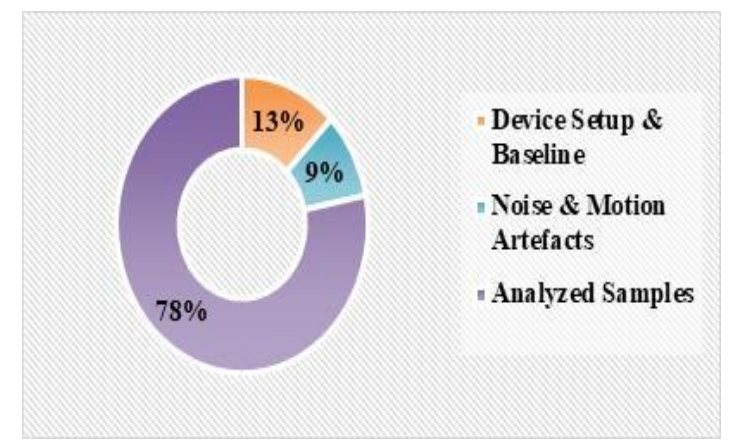

Figure 4. Division of acquired EDA response samples

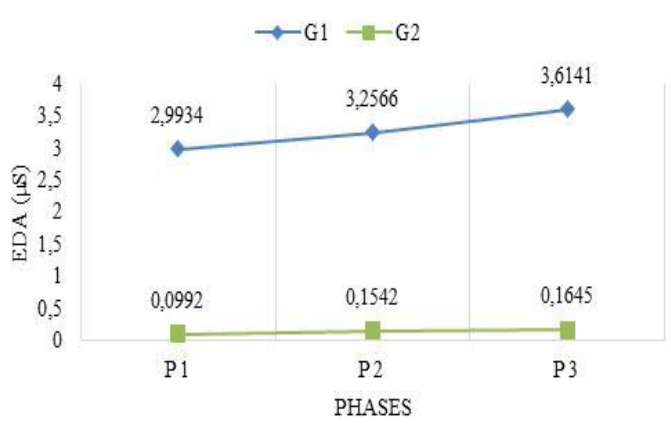

Figure 5. Mean of EDA response signals of G1 and G2

\footnotetext{
Significance of electrodermal activity response in children with autism spectrum disorder (Awais Gul Airij)
} 
Table 1. Mean, variance, and standard deviation of EDA response signals

\begin{tabular}{|c|c|c|c|c|c|c|}
\hline \multirow{2}{*}{ No. } & \multicolumn{3}{|c|}{ G1 } & \multicolumn{3}{|c|}{ G2 } \\
\hline & $\mu$ & $\sigma^{2}$ & $\sigma$ & $\mu$ & $\sigma^{2}$ & $\sigma$ \\
\hline 1 & 3.377 & 1.384 & 1.176 & 2.625 & 0.030 & 0.173 \\
\hline 2 & 6.390 & 2.063 & 1.436 & 1.949 & 0.028 & 0.169 \\
\hline 3 & 10.473 & 7.418 & 2.723 & 11.574 & 0.055 & 0.235 \\
\hline 4 & 12.395 & 1.181 & 1.086 & 3.420 & 0.052 & 0.228 \\
\hline 5 & 3.260 & 1.576 & 1.255 & 2.763 & 0.054 & 0.233 \\
\hline
\end{tabular}

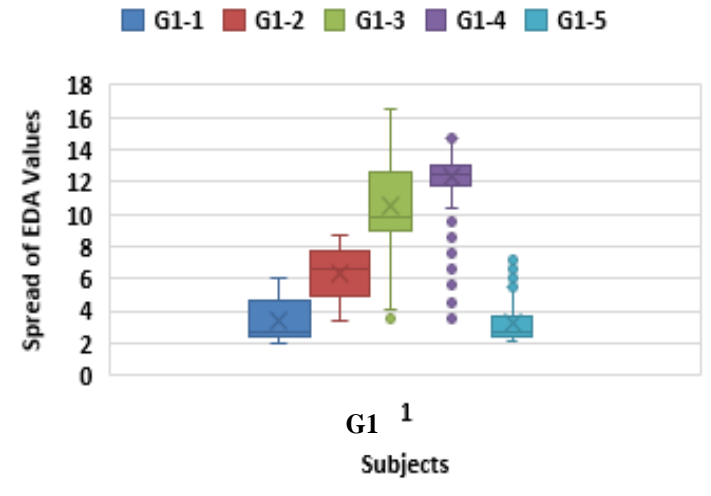

Figure 6. Spread of EDA response values of G1

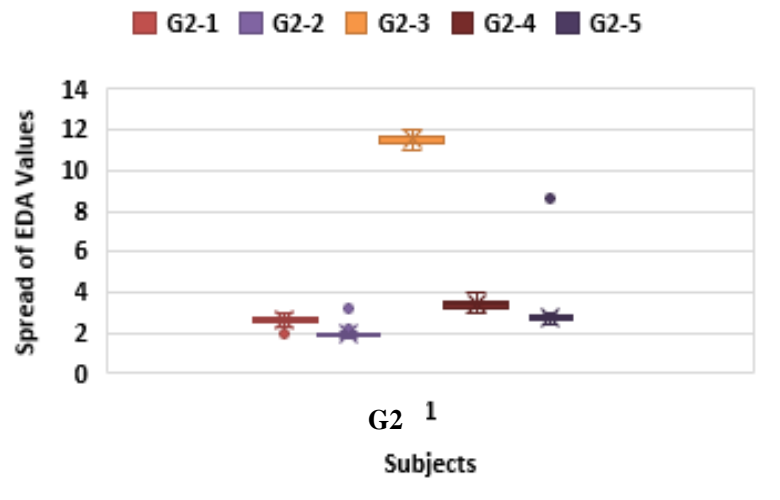

Figure 7. The spread of EDA response values of G2

Moreover, the EDA response signals were classified with the help of implemented SVM classifier and obtained an accuracy of $75 \%$ while using the LF kernel and an accuracy of $66 \%$ was achieved when using RBF kernel. Hence, for this particular study, the LF kernel of SVM classifier provided the best classification results.

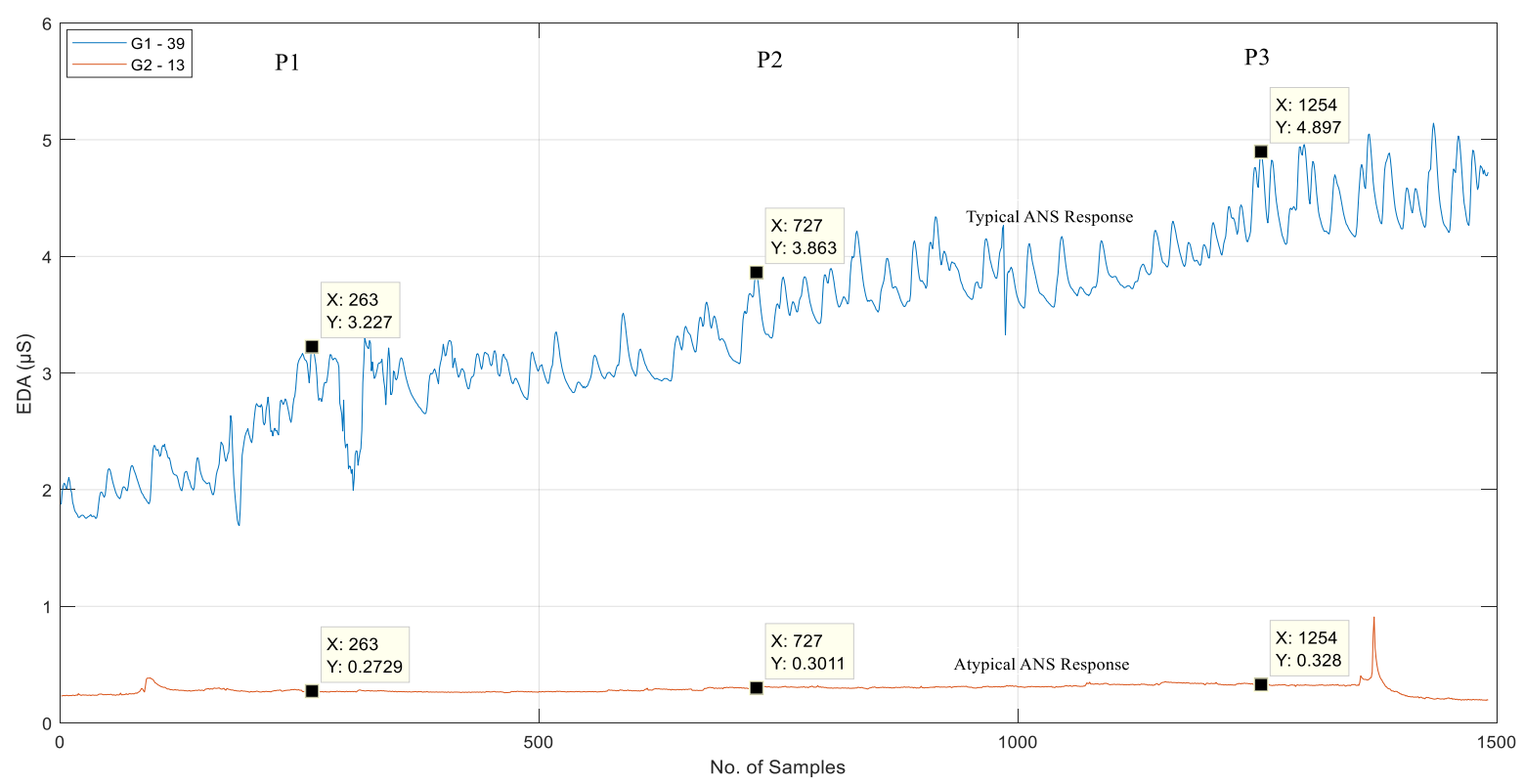

Figure 8. Atypical ANS response of children with ASD

\subsection{Atypical ANS response of children with ASD}

The typical/normal response of a person during the presence of stress is that the physiological signals, including heart rate, skin conductance, and temperature, are increased in magnitude. However, 
in case of children with ASD, they suffer from autonomic dysregulation, therefore they have atypical ANS response. Similar case is highlighted in this research that the obtained samples of EDA response shown in Figure 9 depict that the EDA response of G2 is either blunt or decreases in the presence of a stressor as compared to G1. Such EDA response reflects that the children with ASD have atypical ANS response.

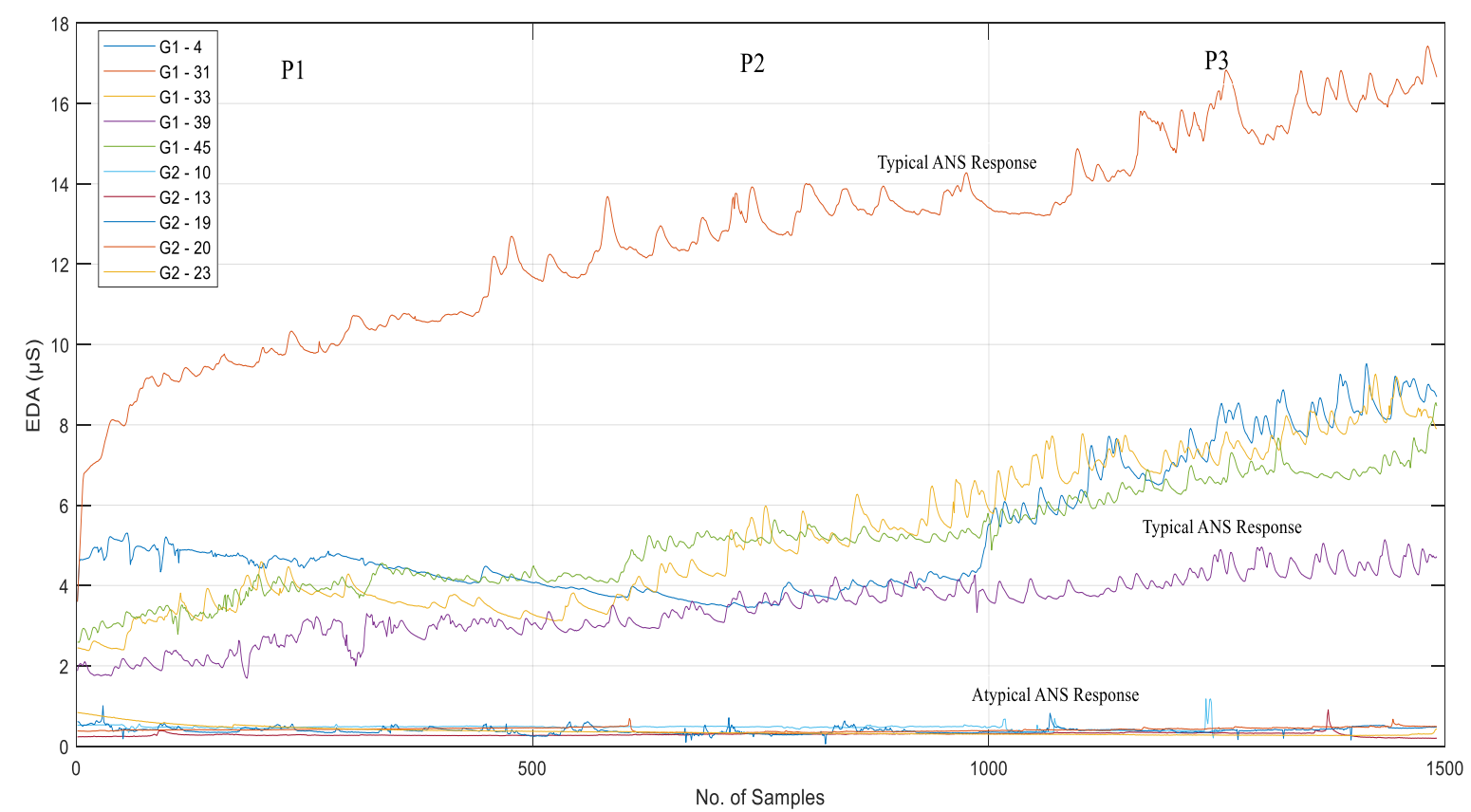

Figure 9. Comparison of ten EDA responses of G1 and G2

\subsection{Discussion}

The SCW test caused a significant increase of tonic and phasic EDA levels in G1, and this effect is in accordance with the regular ANS response to a stressor. This also proves the applicability of the SCW test for inducing stress. However, there were minimal changes in phasic and tonic EDA responses of G2. The obtained results show that children with ASD suffer from autonomic dysregulation. Hence, they depict an atypical ANS response in the presence of stressors. Moreover, the values are shown in Table 1, and the box plots are shown in Figures 6 and 7 also support this as the dispersion of EDA values over time is minimal in case of children with ASD. However, a higher range of values can be witnessed in the EDA values of normal children. Furthermore, the results also support the research [19], stating that the EDA response of children with ASD is blunt or lower as compared to the EDA response of normal children. Hence, resolving the conflict between the two researchers [18, 19] mentioned earlier in Section 1. Finally, the LF kernel of the SVM classifier provided better classification accuracy as compared to the RBF kernel.

\section{CONCLUSION}

This study was aimed at developing an understanding of the sympathetic response of children with ASD in the presence of a stressor. The EDA response datasets were acquired from two groups G1 and G2, where, G1 included 55 normal children and G2 included 35 children with ASD. The obtained results of this study highlight that EDA response is a significant indicator of distress in the case of G1. However, in the case of G2, the results show an atypical ANS response in the presence of a stressor, hence, making the EDA response an insignificant distress marker for children with ASD. This study found a hypoactive sympathetic response of $\mathrm{G} 2$ as opposed to the hyperactive response, which was suggested in previous research. In addition to that, the SVM classifier used for classifying EDA response signals of both groups highlighted that the LF kernel is more suitable for physiological applications when compared to the RBF function as better accuracy of $75 \%$ was achieved by LF. Further research is required to be conducted in this area with a wider dataset. Moreover, along with the EDA response signal, other physiological signals such as heart rate, and temperature also need to be scrutinized. Also, more complex classification algorithms need to be used for achieving better accuracy.

Significance of electrodermal activity response in children with autism spectrum disorder (Awais Gul Airij) 


\section{ACKNOWLEDGEMENTS}

The authors would like to express heartfelt thanks to Ministry of Education, Malaysia and Universiti

Teknologi Malaysia for funding and supporting this research under grant number R.J130000.7851.5F123.

\section{REFERENCES}

[1] G. S. Everly, and J. M. Lating, A Clinical Guide to the Treatment of the Human Stress Response, Third. Springer Berlin Heidelberg, 2012.

[2] D. Girdano and G. S. Everly, Controlling Stress and Tension, 9th ed., Ninth. Pearson, 2013.

[3] M. B. Mohamed, M. Yoshizawa, N. Sugita, S. Yamaki, and K. Ichiji, "Noncontact monitoring of heart rate responses to taste stimuli using a video camera," Indones. J. Electr. Eng. Comput. Sci., vol. 18, no. 1, pp. 293-300, 2019.

[4] A. Fernandes, R. Helawar, R. Lokesh, T. Tari, and A. V. Shahapurkar, "Determination of stress using Blood Pressure and Galvanic Skin Response," in 2014 International Conference on Communication and Network Technologies, 2014, pp. 165-168.

[5] F. Mokhayeri, M. R. Akbarzadeh-T, and S. Toosizadeh, "Mental stress detection using physiological signals based on soft computing techniques," 2011 18th Iran. Conf. Biomed. Eng. ICBME 2011, no. December, pp. 232-237, 2011.

[6] Y. Deng, Z. Wu, C. H. Chu, Q. Zhang, and D. F. Hsu, "Sensor feature selection and combination for stress identification using combinatorial fusion," Int. J. Adv. Robot. Syst., vol. 10, 2013.

[7] F. Bagheri, N. Ghafarnia, and F. Bahrami, "Electrocardiogram (ECG) Signal Modeling and Noise Reduction Using Hopfield Neural Networks," Eng. Technol. Appl. Sci. Res., vol. 3, no. 1, pp. 345-348, 2013.

[8] M. Parellada et al., "The neurobiology of autism spectrum disorders," Eur. Psychiatry, vol. 29, no. 1, pp. 11-19, 2013.

[9] J. Bakker, M. Pechenizkiy, and N. Sidorova, "What's your current stress level? Detection of stress patterns from GSR sensor data," Proc. - IEEE Int. Conf. Data Mining, ICDM, no. 1, pp. 573-580, 2011.

[10] S. Panju, J. Brian, A. Dupuis, E. Anagnostou, and A. Kushki, "Atypical sympathetic arousal in children with autism spectrum disorder and its association with anxiety symptomatology," Mol. Autism, vol. 6, no. 1, pp. 1-10, 2015.

[11] J. J. Braithwaite, D. G. Watson, J. Robert, and R. Mickey, "A Guide for Analysing Electrodermal Activity (EDA) \& Skin Conductance Responses (SCRs) for Psychological Experiments," University of Birmingham, UK, 2013.

[12] C. Hufnagel, P. Chambres, P. R. Bertrand, and F. Dutheil, "The Need for Objective Measures of Stress in Autism," Front. Psychol., vol. 8, no. January, pp. 8-11, 2017.

[13] T. B. Tang, L. W. Yeo, D. Jing, and H. Lau, "Activity Awareness Can Improve Continuous Stress Detection in Galvanic Skin Response," pp. 3-6, 2014.

[14] E. Pittella et al., "Wearable heart rate monitoring as stress response indicator in children with neurodevelopmental disorder," in 2018 IEEE International Symposium on Medical Measurements and Applications (MeMeA), 2018, pp. $1-5$.

[15] V. Markova and T. Ganchev, "Three-step Attribute Selection for Stress Detection based on Physiological Signals," in 2018 IEEE XXVII International Scientific Conference Electronics - ET, 2018, pp. 1-4.

[16] A. G. Airij, R. Sudirman, and U. U. Sheikh, "GSM and GPS based real-time remote physiological signals monitoring and stress levels classification," 2nd Int. Conf. BioSignal Anal. Process. Syst. ICBAPS 2018, pp. 130-135, 2018.

[17] A. Kushki, J. Brian, A. Dupuis, and E. Anagnostou, "Functional autonomic nervous system profile in children with autism spectrum disorder," Mol. Autism, vol. 5, no. 1, pp. 1-10, 2014.

[18] K. C. Welch, "Physiological signals of autistic children can be useful," IEEE Instrum. Meas. Mag., vol. 15, no. 1, pp. 28-32, 2012.

[19] E. Drumm et al., "Investigating the Autonomic Nervous System Response to Anxiety in Children with Autism Spectrum Disorders," PLoS One, vol. 8, no. 4, p. e59730, 2013.

[20] Empatica, "E4 Wristband User's Manual 20150608," Milano, Italy, 2015.

[21] C. McCarthy, N. Pradhan, C. Redpath, and A. Adler, "Validation of the Empatica E4 wristband," 2016 IEEE EMBS Int. Student Conf. Expand. Boundaries Biomed. Eng. Heal. ISC 2016 - Proc., pp. 1-4, 2016.

[22] IMotions, "GSR Pocket Guide The pocket guide," pp. 1-36, 2016.

[23] F. Scarpina and S. Tagini, "The stroop color and word test," Front. Psychol., vol. 8, no. APR, pp. 1-8, 2017.

[24] J. Z. J. Zhai, a. B. Barreto, C. Chin, and C. L. C. Li, "Realization of stress detection using psychophysiological signals for improvement of human-computer interactions," Proceedings. IEEE SoutheastCon, 2005., pp. 415-420, 2005.

[25] R. Pitale, K. Tajane, and J. Umale, "Heart Rate Variability Classification and Feature Extraction Using Support Vector Machine and PCA: An Overview,” J. Eng. Res. Appl. www.ijera.com, vol. 4, no. 1, pp. 381-384, 2014. 